\title{
Erratum
}

\section{EROs found behind lensing clusters}

\section{Stellar populations and dust properties of optical dropout EROs and comparison with related objects}

D. Schaerer ${ }^{1,2}$, A. Hempel ${ }^{1}$, E. Egami ${ }^{3}$, R. Pelló ${ }^{2}$, J. Richard ${ }^{2,4}$, J.-F. Le Borgne ${ }^{2}$, J.-P. Kneib ${ }^{5}$, M. Wise ${ }^{6}$, and F. Boone ${ }^{7}$

1 Geneva Observatory, University of Geneva, 51, chemin des Maillettes, 1290 Sauverny, Switzerland

e-mail: [daniel.schaerer; angela.hempel]@obs.unige.ch

2 Observatoire Midi-Pyrénées, Laboratoire d'Astrophysique, UMR 5572, 14 Avenue E.Belin, 31400 Toulouse, France

3 Steward Observatory, University of Arizona, 933 North Cherry Street, Tucson, AZ 85721, USA

4 Caltech Astronomy, MC105-24, Pasadena, CA 91125, USA

5 OAMP, Laboratoire d'Astrophysique de Marseille, UMR 6110 traverse du Siphon, 13012 Marseille, France

6 Astronomical Institute Anton Pannekoek, Kruislaan 403, 1098 SJ Amsterdam, The Netherlands

7 Observatoire de Paris, LERMA, 61 Av. de l'Observatoire, 75014 Paris, France

A\&A 469, 47-60 (2007), DOI: 10.1051/0004-6361:20077127

Key words. galaxies: high-redshift - cosmology: early Universe - galaxies: evolution - galaxies: starburst - infrared: galaxies errata, addenda

The stellar masses derived from the SED fits using Hyperz and given in Tables 5-8 are overstimated by a factor $(1+z)$. Stellar masses derived using Eq. (1) and other quantities are not affected by this error. 